\title{
Is efficiency of crude oil market affected by multifractality? Evidence from WTI crude oil market
}

\author{
Rongbao $\mathrm{Gu}^{1 *}$, Bing Zhang ${ }^{2}$ \\ ${ }^{1}$ School of Finance, Nanjing University of Finance \& Economics, Nanjing 210046, P. R. China \\ ${ }^{2}$ School of Engineering \& Management, Nanjing University, Nanjing 210093, P. R. China
}

\begin{abstract}
In this paper, we investigate the dynamics of relationship between efficiency and complexity of WTI crude oil market by introducing two indices: efficiency index and multifractality degree. Employing the cross-correlation analysis proposed by Zebende (2010) and the nonlinear Granger causality analysis proposed by Diks and Panchenko (2006), we find that the market efficiency and multifractality of the WTI crude oil returns interact negatively in a nonlinear mechanism. This suggests that the increase of multifractality degree of the WTI crude oil market will lead to the lower degree of market efficiency and vice versa.
\end{abstract}

Keywords: Crude oil market; Efficiency; Multifractality; Nonlinear correlation; Nonlinear Granger causality test JELclassification: E32; G14; Q43

\section{Introduction}

Crude oil plays a significant role in the world economy. Volatility of oil prices is one of the crucial factors affecting the future economic stability and economic growth. Since the oil market is quite complex, many scholars began to search oil markets in the perspective of nonlinearity.

Panas and Ninni (2000) conducted chaos and nonlinear process test for the Rotterdam and Mediterranean crude oil markets using correlation dimension, entropies and Lyapunov exponents and found that there exists strong evidence of chaos in the price series of many oil products. Subsequently, Adrangia et al. (2001) also found the evidence of the presence of nonlinear phenomena in a number of international energy markets including crude oil market.

Alvarez-Ramirez et al. (2002) studied daily records of international crude oil prices using multifractal analysis. This is the first work to explore the dynamics of crude oil markets in the area of econophysics. They found that crude oil market is a persistent process using rescaled range Hurst analysis. Meanwhile, they also found that crude oil market has a multifractal structure using height-height correlation analysis and two characteristic time scales using moving-average-based filtering.

Tabak and Cajueiro (2007) analyzed the efficiency of crude oil markets by means of testing for time-varying degrees of long-range dependence using moving time windows and rescaled range Hurst analysis. They found that the crude oil market has been more and more efficient over time. Moreover, they found that volatility, a proxy by absolute returns, for crude oil prices has highly persistence. This implies that volatility models such as GARCH or EGARCH cannot be used to estimate and forecast crude oil prices volatility and option pricing, as the Black and Scholes model cannot be used to price crude oil options.

Alvarez-Ramirez et al. (2008) analyzed the auto-correlations of international crude oil prices on the basis of the estimation of the Hurst exponent dynamics for returns using mutifractal detrended fluctuation analysis, which reduces the effects of non-stationary market trends and

\footnotetext{
${ }^{*}$ Corresponding author. Email: rbgu@ sina.com
} 
focuses on the intrinsic auto-correlation structure of market volatilities over different time horizons. They found that the crude oil market exhibits a time-varying short-term inefficient behavior but becomes efficient in the long-term.

Based on the work in Tabak and Cajueiro (2007) and Alvarez-Ramirez et al. (2008), Wang and Liu (2010) tested for the efficiency of WTI crude oil market through observing the dynamic of local Hurst exponents employing the method of rolling window based on the multifractal detrended fluctuation analysis. Their results show that short-term, medium-term and long-term behaviors were generally turning into efficient behavior over time. However, in this way, the results also show that the market did not evolve along stable conditions for long times. They also found that the small volatilities of WTI crude oil market were persistent; however, the large volatilities had high instability, both in the short- and long-terms.

Alvarez-Ramirez et al. (2010) studied the efficiency of international crude oil market using lagged detrended fluctuation analysis to detect delay effects in price autocorrelations quantified in terms of a multiscaling Hurst exponent. They found important deviations from efficiency associated to lagged autocorrelations, so imposing the random walk for crude oil prices has pronounced costs for forecasting. Evidences in favor of price reversion to a continuously evolving mean underscores the importance of adequately incorporating delay effects and multiscaling behavior in the modeling of crude oil price dynamics.

Martina et al. (2011), Barkoulas et al. (2012) and Ortiz et al. (2012) also investigated efficiency of international crude oil market using entropy analysis.

With the better insight of nonlinear complex characteristics of financial markets, people begin to pay attention to the association between complex features of financial markets and market efficiency. Zunino et al. (2008) proposed a model to test the relationship between stage of market development and multifractality degree. By testing for the multifractality degree in 32 equity index returns for different countries, they found stock market inefficiency may be negatively associated with multifractality degree, i.e., higher multifractality is associated with a less developed market (or emergent market). The emerging markets have greater correlation than developed markets, suggesting more predictability. Thus, emerging economies seem to be less efficient than developed ones.

Abovementioned studies focus on complex characteristics of international crude oil market from different perspectives, and establish a simple linear regression between some complex features and efficiency for crude oil markets. This can help us to deepen our understanding of complexity of the price movements of international crude oil market. However, what we are more concerned about is the association between complex characteristics and market efficiency of the price operation dynamic system. For example, whether or not the multifractality is related with the market efficiency for crude oil market? If there is relevance, is it nonlinear correlation? Does multifractality affects market efficiency for the crude oil market? If it does, how does it affect the efficiency of crude oil market? In this paper, through the introduction of market efficiency index and multifractlity degree, we will systematically study the association between efficiency and multifractlity degree of international crude oil market from both linear and nonlinear perspectives by the method of rolling windows. To the best of our knowledge, this paper is the first one which reveals the link between efficiency and multifractlity degree of international crude oil market from both linear and nonlinear aspects and thus provides a deep understanding of inner mechanisms of crude oil markets. The exploration of the linkage between multifractlity degree and market 
efficiency of international crude oil market is a topic worthy of careful study.

The remainder of this paper is organized as follows: Section 2 presents the market efficiency index and the concept of multifractality degree; Section 3 describes the methods of nonlinear correlation analysis and nonlinear causality analysis; Section 4 is a description of the data, the computation and basic analysis of indices; Section 5 presents the linear and nonlinear correlation analysis of efficiency index and multifractality degree for the WTI crude oil market, and section 6 conducts the linear and nonlinear causality analysis between them. The final section gives a brief conclusion.

\section{Choice of indices}

To establish the efficiency index and the multifractality degree for crude oil market, in this section, we introduce multifractal detrended fluctuation analysis and give their explanations on finance.

\subsection{Efficiency index}

According to the Efficiency Market Hypothesis which is introduced by Fama (1970), the efficient markets have three levels, including weak-form efficiency, semi-strong-form efficiency, and strong-form efficiency. A market is deemed as weak-form efficiency if the asset prices can reflect all historical information. A market is semi-strong-form efficiency if the asset prices can reflect not only all historical information but also all public information. A market is strong-form efficiency if the asset prices can reflect not only all historical information and all pubic information but all insider information. If a market is weak-form efficiency, all historical information will be included in the current prices and the prices will follow a random walk.

The Hurst exponent, posed by Hurst (1951) in studying Lino River, is an important scale which can be used to test whether a time series follows a random walk. A robust way to calculate Hurst exponent is the detrended fluctuation analysis (DFA) proposed by Peng et al. (1994). The DFA procedure consists of five steps as follows:

Step 1. Let $\left\{x_{t}, t=1, \cdots, N\right\}$ be a time series, where $N$ is the length of the series. Determine the "profile"

$$
x x_{k}=\sum_{t=1}^{k}\left(x_{t}-\bar{x}\right), \quad k=1,2, \cdots, N
$$

where $\bar{x}$ denotes the averaging over the whole time series.

Step 2. Divide the profile $\left\{x x_{k}\right\}_{k=1, \cdots, N}$ into $N_{s} \equiv \operatorname{int}(N / s)$ non-overlapping segments of equal length $s$. Since the length $N$ of the series is often not a multiple of the considered time scale $s$, a short part at the end of the profile may remain. In order not to disregard this part of the series, the same procedure is repeated starting from the opposite end. Thereby, $2 N_{s}$ segments are obtained altogether. Introduced by Peng et al. (1994), we get $5 \leq s \leq N / 5$.

Step 3. Calculate the local trend for each of the $2 N_{s}$ segments by a least-square fit of the series. Then determine the variance 


$$
F^{2}(s, \lambda) \equiv \frac{1}{s} \sum_{j=1}^{s}\left[x x_{(\lambda-1) s+j}-P_{\lambda}(j)\right]^{2}
$$

for $\lambda=1,2, \cdots, N_{s}$ and

$$
F^{2}(s, \lambda) \equiv \frac{1}{s} \sum_{j=1}^{s}\left[x x_{N-\left(\lambda-N_{s}\right) s+j}-P_{\lambda}(j)\right]^{2}
$$

for $\lambda=N_{s}+1, N_{s}+2, \cdots, 2 N_{s}$. Here, $P_{\lambda}(j)$ is the fitting polynomial with order $m$ in segment $\lambda$.

Step 4 . Average over all segments to obtain the volatility function

$$
F(s)=\left\{\frac{1}{2 N_{s}} \sum_{\lambda=1}^{2 N_{1}} F^{2}(s, \lambda)\right\}^{1 / 2} .
$$

We repeat steps 2 to 4 several time scales $s$. It is apparent that $F(s)$ will increase with increasing $s$. Of course, $F(s)$ depends on the DFA order $m$. By construction, $F(s)$ is only defined for $s \geq m+2$.

Step 5. Determine the scaling behavior of the volatility functions by analyzing log-log plots $F(s)$ versus $s$. For large values of $s$, as a power-law,

$$
F(s) \sim s^{H}
$$

where $H$ is called the Hurst exponent, which can be obtained by observing the slope of log-log plots of $F(s)$ versus $s$ through the method of least square.

If $H>0.5$, the time series is persistent or long-range dependence. If $H<0.5$, the time series is anti-persistent or mean recurrence. If $H=0.5$, the time series displays random walk behavior.

For a financial market, if the Hurst exponent of the asset prices or returns is more close to 0.5, the market will be more close to weak-form efficiency. Many authors use the Hurst exponent to measure the level of efficiency of various financial markets, such as, Tabak and Cajueiro (2007), Alvarez-Ramirez et al. (2008), Zunino et al. (2008) and Wang et al. (2010), etc. For convenience, we introduce the market efficiency index as

$$
E I=|H-0.5|
$$

This means that, the smaller the $E I$ value of a market is, the higher the efficiency of the market could be.

\subsection{Mutifractality degree}

Kantelhardt et al. (2002) proposed the multifractal detrended fluctuation analysis (MF-DFA), which is a generalization of the DFA. The MF-DFA can be used for a global detection of multifractal behavior of a non-stationary time series. The MF-DFA procedure also consists of five steps as follows:

Let $\left\{x_{t}, t=1, \cdots, N\right\}$ be a time series. The first three steps are just the same as the DFA method.

Step 4. Average over all segments to obtain the $q$ th order volatility function

$$
F_{q}(s)=\left\{\frac{1}{2 N_{s}} \sum_{\lambda=1}^{2 N_{s}}\left[F^{2}(s, \lambda)\right]^{q / 2}\right\}^{1 / q}
$$


for any real value $q \neq 0$ and

$$
F_{0}(s)=\exp \left\{\frac{1}{4 N_{s}} \sum_{\lambda=1}^{2 N_{s}} \ln \left[F^{2}(s, \lambda)\right]\right\} .
$$

We repeat steps 2 to 4 several time scales $s$. It is apparent that $F_{q}(s)$ will increase with increasing $s$. By construction, $F_{q}(s)$ is only defined for $s \geq m+2$.

Step 5. Determine the scaling behavior of the volatility functions by analyzing log-log plots $F_{q}(s)$ versus $s$ for each value of $q$. For large values of $s$, as a power-law,

$$
F_{q}(s) \sim s^{h(q)}
$$

where $h(q)$ is called the generalized Hurst exponent with order $q$, which can be obtained by observing the slope of $\log$-log plots of $F_{q}(s)$ versus $s$ through the method of least square.

The time series is called to be multifractal if $h(q)$ depends on $q$ and monofractal if $h(q)$ is independent of $q$. A mutifractal series has a structure that is relatively more complicated than that of a monofractal series.

The generalized Hurst exponents not only describe the macroscopic appearance of sequence volatility, but also give more description on some features of sequence volatility. For positive values of $q, h(q)$ describes the scaling behavior of the segments with large volatilities, and for negative values of $q, h(q)$ describes the scaling behavior of the segments with small volatilities. Hence, the bigger the spread domain of generalized Hurst exponent $h(q)$ is, the more complicated the structure of the series is.

Zunino et al. (2008) proposed the multifractality degree as

$$
\Delta h=h\left(q_{\min }\right)-h\left(q_{\max }\right)
$$

and suggested that the inefficiency of market can be measured by multifractality degree of the price returns. However, this definition is not appropriate to some financial markets ( $\mathrm{Gu}$ et al., 2013). The multifractality degree should be modified as

$$
M D=\max _{q} h(q)-\min _{q} h(q) .
$$

For a financial market, the higher the $M D$ value of a market is, the more complicated the market could be.

It is pointed by Kantelhardt et al. (2002) that $h(2)$ is identical to the well-known Hurst exponent $H$ for a stationary time series. Therefore, running the MF-DFA on a financial market we can obtain not only the information of the market multifractality but the message of the market efficiency.

It is worth noting that the Hurst exponent which is used to define the market efficiency index series is fortunately as a member $(h(2))$ of the generalized Hurst index $h(q)$, then whether the market efficiency index series and multifractlity degree exist some kind of mathematical inevitable connection? We haven't seen any mathematical proof of this in so far. In fact, Gu et al. (2013) had explored this issue, the static analysis of 34 international stock markets as well as the dynamic analysis of the Shanghai stock market itself all showed that there is no simple linear 
correlation between market efficiency index series and multifractlity degree series, but can be demonstrated different nonlinear correlations in different time intervals. Because of this, the exploration of the association between multifractlity degree and market efficiency of international crude oil market is a topic worthy of study.

\section{Methodology}

\subsection{Nonlinear correlation coefficient}

The most popular measure of correlation is Pearson correlation coefficient. However, this coefficient is not robust and can be misleading if outliers are present. Moreover, this coefficient is also misleading for the series with non-stationarity or non-Gaussian distribution. Recently, Zebende (2010) proposed a new cross-correlation coefficient in order to quantify the level of cross-correlation between two series with non-stationary and non-Gaussian distribution. This algorithm of correlation coefficients can be used to measure the correlation between two non-stationary series for the different time scales. The steps of the algorithm can be described as follows.

Step 1. Consider two time series, $\left\{x_{t}, t=1,2, \cdots, N\right\}$ and $\left\{y_{t}, t=1,2, \cdots, N\right\}$, where $N$ is the equal length of these two series. Then, we describe the "profile" of each series and get two new series

$$
x x_{k}=\sum_{t=1}^{k}\left(x_{t}-\bar{x}\right) \text { and } y y_{k}=\sum_{t=1}^{k}\left(y_{t}-\bar{y}\right), k=1,2, \cdots, N .
$$

Step 2. Divide the both profiles $\left\{x x_{k}\right\}$ and $\left\{y y_{k}\right\}$ into $N_{s} \equiv \operatorname{int}(N / s)$ nonoverlapping segments of equal length $s$. Since the length $N$ of the series is often not a multiple of the considered time scale s, a short part at the end of each profile may remain. In order not to disregard this part of the series, the same procedure is repeated starting from the opposite end of each profile. Thereby, $2 N_{s}$ segments are obtained together. We set $5 \leq s \leq N / 5$, introduced by Peng et al. (1994).

Step 3. Calculate the local trends for each of the $2 N_{s}$ segments by a least-square fit of each series. Then determine the covariance

$$
F_{x y}(s, \lambda)=\frac{1}{s} \sum_{j=1}^{s}\left[x x_{(\lambda-1) s+j}-x P_{\lambda}(j)\right]\left[y y_{(\lambda-1) s+j}-y P_{\lambda}(j)\right]
$$

for $\lambda=1,2, \cdots, N_{s}$ and

$$
F_{x y}(s, \lambda)=\frac{1}{s} \sum_{j=1}^{s}\left[x x_{N_{s}-\left(\lambda-N_{s}\right) s+j}-x P_{\lambda}(j)\right]\left[y y_{N_{s}-\left(\lambda-N_{s}\right) s+j}-y P_{\lambda}(j)\right]
$$

for $\lambda=N_{s}+1, N_{s}+2, \cdots, 2 N_{s}$. Here, $x P_{\lambda}(j)$ and $y P_{\lambda}(j)$ are the fitting polynomials with order $m$ in segment $\lambda$ of $\left\{x x_{k}\right\}$ and $\left\{y y_{k}\right\}$, respectively.

Step 4. Average over all segments to get the detrended covariance function 


$$
F_{x y}^{2}(s)=\frac{1}{2 N_{s}} \sum_{\lambda=1}^{2 N_{s}} F_{x y}(s, \lambda) .
$$

Step 5. Calculate the DCCA cross-correlation coefficient by using the ratio between the detrended covariance function and the detrended mean variance functions, i.e.,

$$
\sigma_{D C C A}(s)=\frac{F_{x y}^{2}(s)}{F_{x x}(s) F_{y y}(s)} .
$$

Obviously, this nonlinear correlation coefficient depends on the length of each part, $s$, just the time scale in the scaling analysis. So, the outstanding advantage of this coefficient is that it can measure the correlations between two financial time series with the different time scales. The coefficient $\sigma_{D C C A}$ ranges between -1 and 1 . Two series are cross correlated positively if $\sigma_{D C C A}>0$ and negatively if $\sigma_{D C C A}<0$. A value of $\sigma_{D C C A}=0$ means there is no cross correlation between the two series.

\subsection{Nonlinear Granger causality test}

Granger causality has turned out to be a useful notion for characterizing dependence relations between time series in economics and econometrics. We adopt the following VAR model to test for (linear) Granger causality:

$$
\begin{aligned}
& x_{t}=c_{1}+\sum_{i=1}^{m} \alpha_{1 i} x_{t-i}+\sum_{i=1}^{m} \beta_{1 i} y_{t-i}+\varepsilon_{1 t} \\
& y_{t}=c_{2}+\sum_{i=1}^{m} \alpha_{2 i} x_{t-i}+\sum_{i=1}^{m} \beta_{2 i} y_{t-i}+\varepsilon_{2 t}
\end{aligned}
$$

where $\varepsilon_{1 t}$ and $\varepsilon_{2 t}$ are two error terms, $m$ is the optimal lag length obtained by using the Schwartz Information Criterion (SIC). The null hypothesis that $y_{t}$ does not Granger cause $x_{t}$ is equivalent to testing $\beta_{1 i}=0$ for all $i=1,2, \cdots, m$ in Eq. (17).

The linear Granger causality test does not account for nonlinear causality relationships among the variables. In order to test for nonlinear Granger causality, various nonparametric methods are developed. In an early study, Baek and Brock (1992) proposed a nonparametric statistical method for detecting nonlinear Granger causality employing correlation integral between time series. In Baek and Brock's test, the time series are as assumed to be mutually and individually independent and identically distributed. By relaxing this strict assumption, Hiemstra and Jones (1994) developed a modified test statistic for the nonlinear causality, which allows search series to display short-term dependence. However, Diks and Panchenko (2006) showed that the test advocated by Hiemstra and Jones (1994) may over reject the null hypothesis of non-causality in the case of increasing sample size since it ignores the possible variation in conditional distributions. Diks and Panchenko (2006) developed a new nonparametric test for Granger causality that overcomes the over-rejection problem in the Hiemstra and Jones test.

For stationary time series $\left\{x_{t}\right\}$ and $\left\{y_{t}\right\}$, we suppose delay vectors $X_{t}^{\ell_{x}}=\left(x_{t-\ell_{x}+1}, \cdots, x_{t}\right)$ $Y_{t}^{\ell}=\left(y_{t-\ell_{y}+1}, \cdots, y_{t}\right)\left(\ell_{x}, \ell_{y} \geq 1\right)$. The null hypothesis that past observations of $X_{t}^{\ell x}$ contain no additional information (beyond that in $Y_{t}^{\ell}{ }^{y}$ ) about $y_{t+1}$ is tested, i. e., 


$$
H_{0}: y_{t+1}\left|\left(X_{t}^{\ell_{x}} ; Y_{t}^{\ell_{y}}\right) \sim y_{t+1}\right| Y_{t}^{\ell_{y}} .
$$

The null hypothesis becomes a statement about the invariant distribution of the $\left(\ell_{x}+\ell_{y}+1\right)$ dimensional vector $W_{t}=\left(X_{t}^{\ell_{x}}, Y_{t}^{\ell_{y}}, z_{t}\right)$, where $z_{t}=y_{t+1}$. If we ignore the time index and we assume that $\ell_{x}=\ell_{y}=1$, the distribution of $Z$ given that $(x, y)=(u, v)$ is the same as that of $z$ given $y=v$. In that case, Eq. (19) is restructured to take into account the ratios of joint distributions. In that sense, the joint probability density function $f_{x, y, z}(u, v, w)$ and its marginal density functions should satisfy the following relationship:

$$
\frac{f_{x, y, z}(u, v, w)}{f_{y}(v)}=\frac{f_{x, y}(u, v)}{f_{y}(v)} \cdot \frac{f_{y, z}(v, w)}{f_{y}(v)} .
$$

In other words, Eq. (20) states that $x$ and $z$ are independent when $y=v$ for each fixed value of $v$. Diks and Panchenko (2006) show that the restated null hypothesis implies:

$$
q \equiv E\left[f_{x, y, z}(x, y, z) f_{y}(y)-f_{x, y}(x, y) f_{y, z}(y, z)\right]=0 .
$$

Suppose $\hat{f}_{W}\left(W_{i}\right)$ is a local density estimator of a $d_{W}$-variate random vector $W$ at $W_{i}$, defined by $\hat{f}_{W}\left(W_{i}\right)=\left(2 \varepsilon_{n}\right)^{-d_{W}}(n-1)^{-1} \sum_{j: j \neq i} I_{i j}^{W}$ where $I_{i j}^{W}=I\left(\left\|W_{i}-W_{j}\right\|<\varepsilon_{n}\right)$, with $I(\cdot)$ the indicator function and $\varepsilon_{n}$ the bandwidth, depending on the sample size $n$. Then, the test statistic is a scaled sample version of $q$ in Eq. (21):

$$
T_{n}\left(\varepsilon_{n}\right)=\frac{n-1}{n(n-2)} \sum_{i}\left(\hat{f}_{x, y, z}\left(x_{i}, z_{i}, y_{i}\right) \hat{f}_{y}\left(y_{i}\right)-\hat{f}_{x, y}\left(x_{i}, y_{i}\right) \hat{f}_{y, z}\left(y_{i}, z_{i}\right)\right) .
$$

For $\ell_{x}=\ell_{y}=1$, if $\varepsilon_{n}=C n^{-\beta}\left(C>0, \frac{1}{4}<\beta<\frac{1}{3}\right)$ then Diks and Panchenko (2006) prove under strong mixing that the test statistic in Eq. (22) satisfies:

$$
\sqrt{n} \frac{\left(T_{n}\left(\varepsilon_{n}\right)-q\right)}{S_{n}} \stackrel{D}{\longrightarrow} N(0,1)
$$

where $\stackrel{D}{\longrightarrow}$ denotes convergence in distribution and $S_{n}$ is an estimator of the asymptotic variance of $T_{n}(\cdot)$. We followed Diks and Panchenko's suggestion to implement a one-tailed version of the test, rejecting the null hypothesis if the left-hand-side of Eq. (23) is too large.

\section{Data description and preliminary analysis on indices}

The data of the study consist of the daily closing spot prices for the West Texas Intermediate (WTI) and are given in US dollar per barrel. The data span from January 2, 1986 to September 28, 2012, namely 6846 observations. The dataset is extracted from the Energy Information Administration (EIA) in the U.S. Department of Energy. 
Let $p_{t}$ be the price of crude oil on day $t$. The daily price returns, $r_{t}$, are calculated as its logarithmic difference,

$$
r_{t}=\log \left(p_{t} / p_{t-1}\right)
$$

Fig. 1 and Fig. 2 provide a graphical representation of these series, respectively.

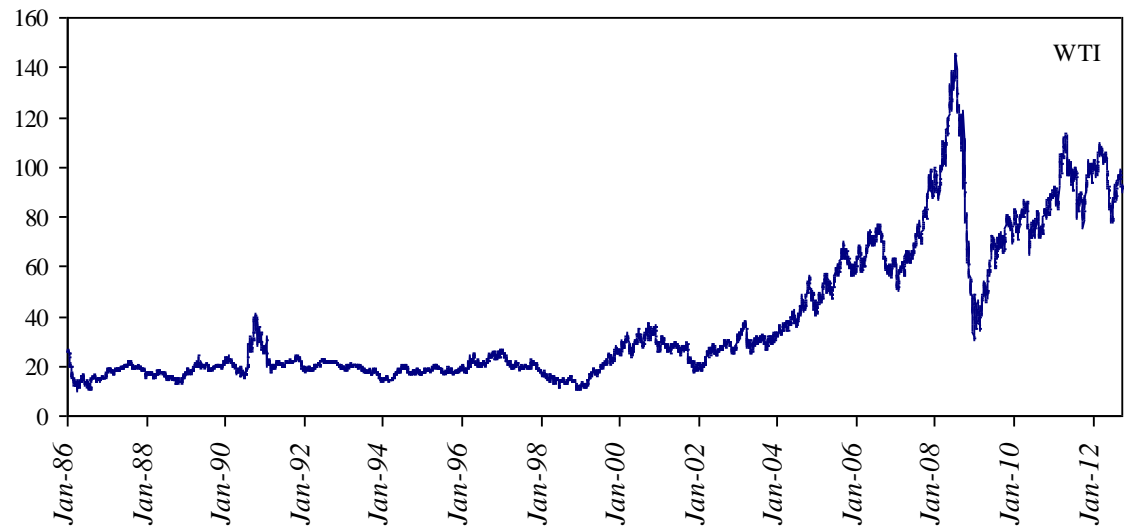

Fig. 1. Daily WTI crude oil prices.

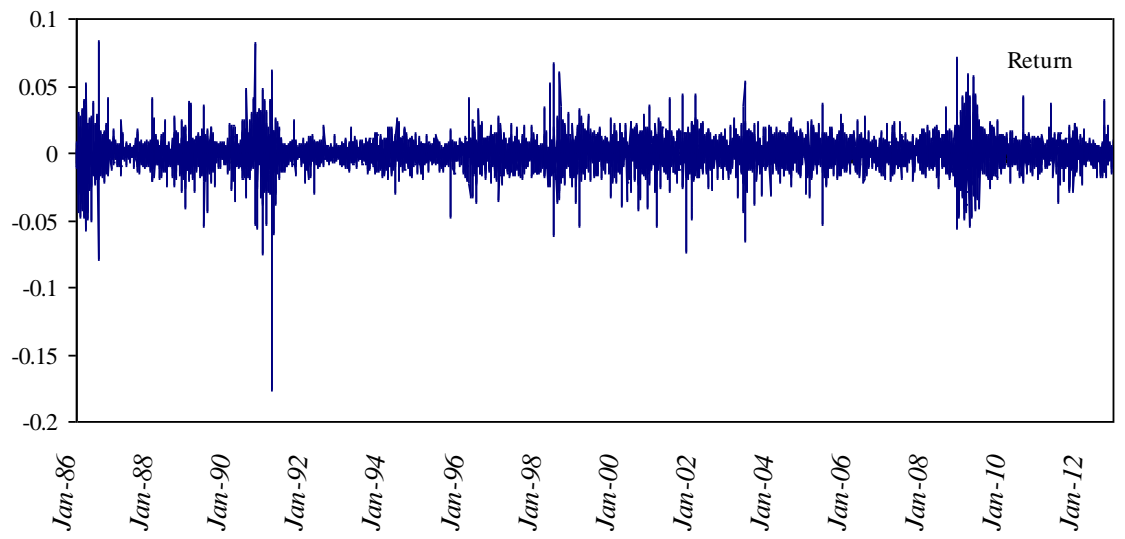

Fig. 2. Daily WTI crude oil returns.

To study the dynamics of efficiency and multifractality of WTI crude oil market, we estimate the time-varying generalized Hurst exponents for a 4 year (1008 observations) time-window (Tabak and Cajueiro (2007)) and a 1 year (250 observations) time-window (Wang et al. (2010)) respectively. The data in the $x$-axis stand for the ending of the sample used in the estimation of the Hurst exponents. Therefore, for a data Jan-97 the Hurst exponent were evaluated for the sample beginning 4 year before (Jan-93) and ending in Jan-97 for moving windows with fixed length of 1008 observations and so forth.

We denote by $E I 4$ and $E I 1$ the efficient indices of returns with 4 year time-window and 1 year time-window respectively, by $M D 4$ and $M D 1$ the multifractality degrees of returns with 4 year time window and 1 year window respectively. Fig. 3 and Fig. 4 provide a graphical representation of these series in different time-windows. respectively. 


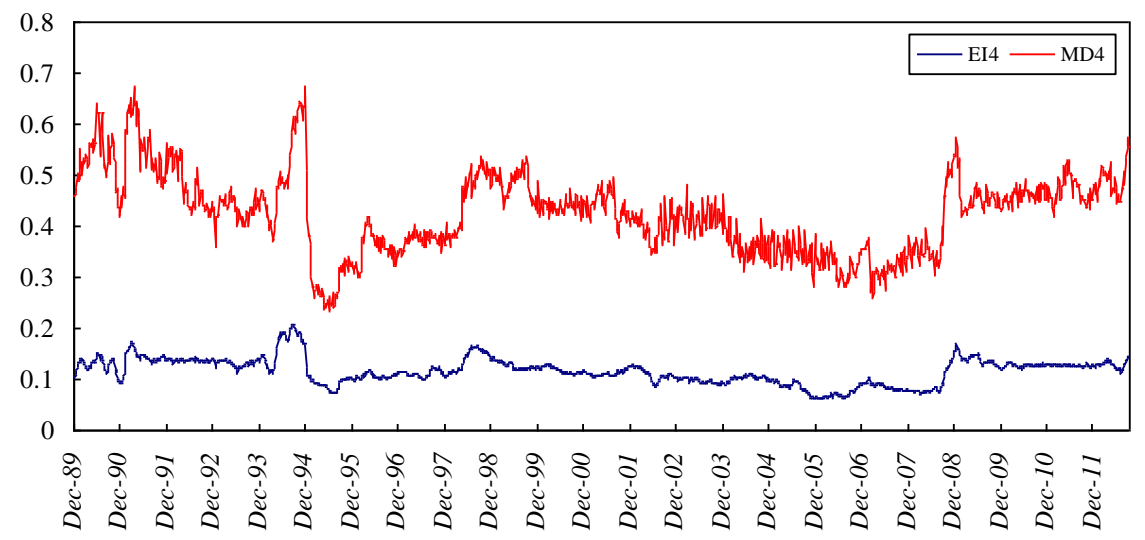

Fig. 3. Time-varying efficiency indices and multifractality degrees for 4 year time-window.

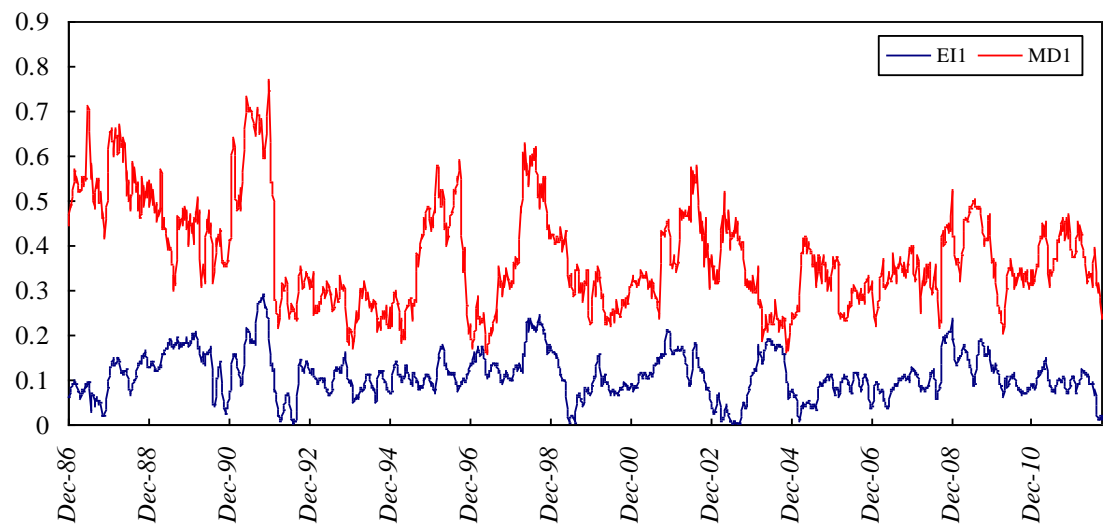

Fig. 4. Time-varying efficiency indices and multifractality degrees for 1 year time-window.

Table 1 reports a descriptive statistic for efficiency indices and multifractality degrees of the WTI crude oil returns in different time-windows.

Table 1. Descriptive statistics.

\begin{tabular}{ccccc}
\hline & $E I 4$ & $M D 4$ & $E I 1$ & $M D 1$ \\
\hline Mean & 0.1162 & 0.4251 & 0.1111 & 0.3773 \\
Std. Dev. & 0.0253 & 0.0795 & 0.0503 & 0.1166 \\
Skewness & 0.3050 & 0.2391 & 0.4141 & 0.6924 \\
Kurtosis & 3.5087 & 2.9847 & 3.4049 & 3.0316 \\
Jarque-Bera & 30.682 & 11.133 & 46.749 & 105.53 \\
Probability & 0.0000 & 0.0000 & 0.0000 & 0.0000 \\
\hline
\end{tabular}

As Table 1 shows, whether $E I$ or $M D$, the average level for 4 year time-window is larger than that for 1 year time-window, but the standard deviation for 1 year time-window is larger than that for 4 year time-window. The skewness of all index series are greater than 0.2 , and skewness for 1 year time-window are greater than that for 4 year time-window, showing that all series are left deviation and skewness for 1 year time-window are greater. The J-B statistic tests for each index 
series show that all index series do not follow normal distribution.

In order to study the stationary of efficiency index and multifractlity degree of the WTI crude oil returns, we use the ADF and PP unit root tests in these series and the results are given in Table 2 .

Table 2. Unit root test.

\begin{tabular}{ccccc}
\hline & \multicolumn{2}{c}{ ADF test } & \multicolumn{2}{c}{ PP test } \\
\cline { 2 - 5 } & $t$-statistic & Probability & $t$-statistic & Probability \\
\hline$E I 4$ & -3.4538 & 0.0095 & -3.3722 & 0.0122 \\
$M D 4$ & -3.1441 & 0.0237 & -5.0750 & 0.0000 \\
$E I 1$ & -5.5002 & 0.0000 & -5.0582 & 0.0000 \\
$M D 1$ & -4.3962 & 0.0003 & -4.4588 & 0.0002 \\
\hline
\end{tabular}

As shown in Table 2, EI4 rejects the null hypothesis of unit root under the 5\% significance level for PP test and MD4 does the same for ADF test, and all other series do the same under the $1 \%$ significance level. This suggests that all series are stationary.

\section{Correlation analysis}

The correlation test is divided into three steps. First, we should calculate the Pearson correlation coefficient between efficiency indices and multifractality degrees to test their linear correlation, then calculate the DCCA cross correlation coefficient between two series using the method proposed by Zebende (2010) to test their nonlinear correlation, at last, recalculate the DCCA cross correlation coefficient between two VAR-filtered residuals of original series to confirm that if there are any nonlinear correlations between them.

\subsection{Linear correlation test}

Pearson correlation coefficient is the most popular measure of correlation. Table 2 presents the Pearson correlation coefficients between efficiency index and multifractality degree of the WTI crude oil returns for different time windows. Fig.5 shows scatters of efficiency index and multifraclity degree for different time windows.

Table 3. Pearson correlation coefficient.

\begin{tabular}{|c|c|c|}
\hline Pair & $M D 4$ & $E I 1 \quad M D 1$ \\
\hline Pearson correlation coefficient & 0.8067 & 0.3732 \\
\hline
\end{tabular}

As shown in Table 3, Pearson correlation coefficient between EI4 and MD4 is up to 0.8, and shows strong positive linear correlation, while the Pearson correlation coefficient between $E I 1$ and $M D 1$ is 0.3732 with a weak positive linear correlation.

Scatters in Fig.5 intuitively describe the correlations between efficiency index and multifractlity degree of returns for different time windows, respectively. 

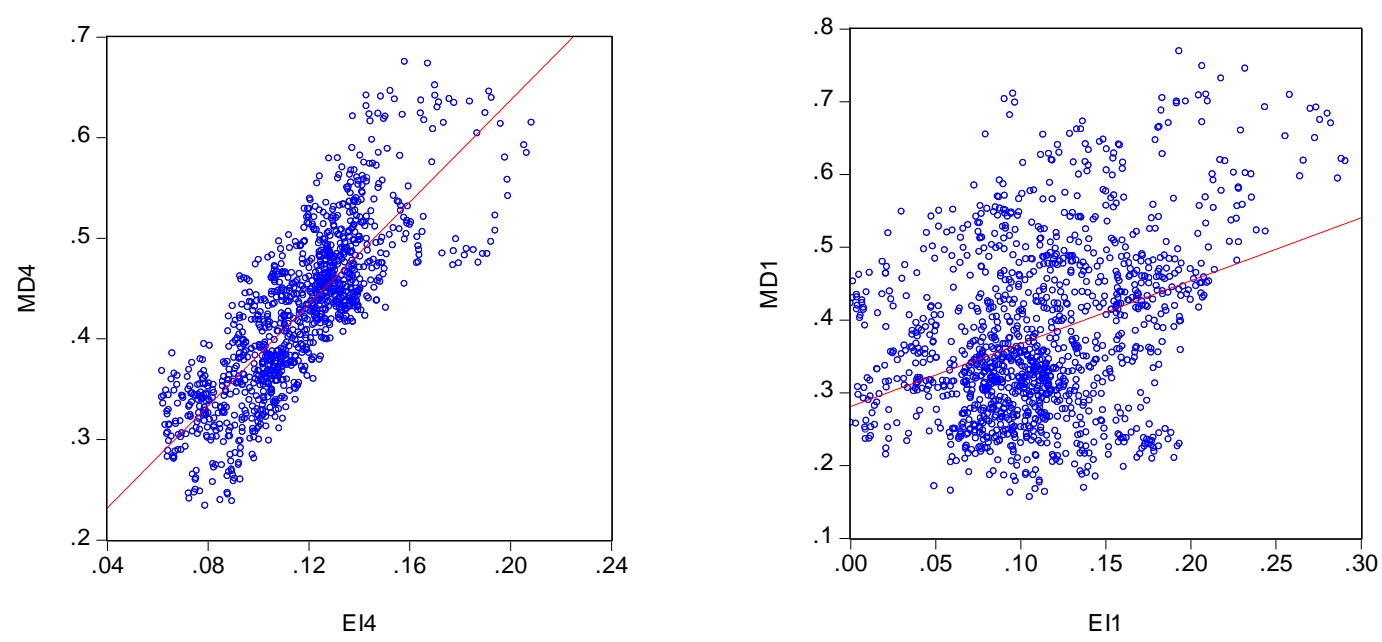

Fig. 5. Scatters of efficiency index and multifractality degree.

\subsection{Nonlinear correlation test for original series}

It can only explain there is no linear correlation between two series if they are not related, but there may be a nonlinear correlation. Therefore, we should test if there is nonlinear correlation between efficiency index and multifractlity degree of the WTI crude oil returns and volatilities. We calculate the DCCA cross correlation coefficients for raw series and filtered VAR-residuals respectively, and the results are reported in the corresponding panels of Table 4.

Table 4. DCCA cross correlation coefficient.

\begin{tabular}{|c|c|c|c|c|c|c|c|}
\hline \multirow{2}{*}{\multicolumn{2}{|c|}{ Pair }} & \multicolumn{6}{|c|}{ DCCA cross correlation coefficient } \\
\hline & & \multicolumn{3}{|c|}{ Raw data } & \multicolumn{3}{|c|}{ VAR } \\
\hline \multirow{2}{*}{ EI4 } & \multirow{2}{*}{$M D 4$} & $5<s \leq 200$ & $200<s \leq 500$ & $500<s$ & $5<s \leq 200$ & $200<s \leq 500$ & $500<s$ \\
\hline & & $0.4 .8 \sim 0.85$ & $0.63 \sim 0.83$ & $0.82 \sim 0.89$ & $0.4 \sim 0.63$ & $0.26 \sim 0.65$ & $0.5 \sim 0.68$ \\
\hline \multirow{2}{*}{ EI1 } & \multirow{2}{*}{$M D 1$} & $5<s \leq 360$ & $360<s \leq 600$ & $600<s$ & $5<s \leq 360$ & $360<s \leq 600$ & $600<s$ \\
\hline & & $0.13 \sim 0.61$ & $0.4 \sim 0.63$ & $0.5 \sim 0.7$ & $0.22 \sim 0.55$ & $0.4 \sim 0.59$ & $0.53 \sim 0.68$ \\
\hline
\end{tabular}

Note: (1) The first line is the range of time scales and the second is the interval of the DCCA cross-correlation coefficients. (2) The lag lengths of VAR are chosen by the Schwarz information criterion (SCI).

The first half of Table 4 shows the DCCA cross correlation coefficient between efficiency index and multifractlity degree. By the partial results, we can see there is a positive nonlinear correlation between $E I$ and $M D$, whether for 4 year time-window or 1 year time-window. The correlations exhibit moderate intensity in the medium term, but in the long run, the performance is stronger.

According to the Pearson correlation coefficients, we found there were strong linear correlations between efficiency index and multifractlity degree of the WTI crude oil returns. Here we found evidences of nonlinear correlations between efficiency index and multifractlity degree of returns by using Zebende's DCCA cross correlation coefficient, among them, for 4 year time-window, the nonlinear and linear correlations between efficiency index and multifractlity degree of returns show high intensity correlation. 


\subsection{Nonlinear correlation testing on VAR-filtered residuals}

The results from the previous two steps suggest that there are some linear and nonlinear correlations between efficiency index and multifractality degree. However, even though we found nonlinear correlations, the DCCA cross correlated coefficient should be recalculated to filtered VAR-residuals to ensure that any correlation is strictly nonlinear in nature.

Because all series are stationary, we establish VAR model to filter $E I$ and $M D$. The lag lengths of the VAR specification where based on the Schwarz information criterion (SIC). The second half of Table 4 shows the DCCA cross correlation coefficient between two VAR-filtered residuals of efficiency index and multifractality degree. This partial results show that there is a positive nonlinear correlation between $E I$ and $M D$, whether for 4 year time-window or 1 year time-window. The correlations also perform somewhat weaker in the short term, while slightly enhance in the long run.

Compare the two partial results in Table 4, we found that the nonlinear correlation test results of $E I 1$ and $M D 1$ are roughly equivalent before and after filtered by VAR, while the nonlinear correlation between EI4 and MD4 filtered by VAR significantly weakened, this is because the original series of the former does not exist linear correlation, while there is a strong linear correlation between the original series of the latter. Nonlinear correlation test on the original series with linear correlations will be affected by linear correlation interference, only after filtering the linear correlation of original series can the inspection results truly reflect the nonlinear correlations between efficiency index and multifractality degree.

In summary, whether for 4 year time-window or 1 year time-window, the test results of efficiency index and multifractality degree of returns show that there always exist nonlinear correlation in different time scales.

\section{Granger causality analysis}

The causality test is also divided into three steps. First, we conduct linear causality test between efficiency index and multifractality degree using the traditional Granger causality test, then use the method proposed by Diks and Panchenko (2006) to do nonlinear causality test between two series, at last, redo Diks-Panchenko nonlinear causality test between two VARfiltered residuals of original series to ensure the authenticity of nonlinear causality.

\subsection{Linear Granger causality test}

The first part of Table 5 shows the results of traditional Granger causality test between efficiency index and multifractality degree of the WTI crude oil returns. From these results, we can see that, there exist bidirectional Granger causalities between EI4 and MD4, but there is no Granger causality in any direction between $E I 1$ and $M D 1$.

The traditional Granger causality tests only detect the presence of linear causality between series. By the discussion of section 5, even if there is no linear correlation between the series, there may be nonlinear correlations, so it is necessary to conduct nonlinear Granger causality test between $E I$ and $M D$. 


\subsection{Nonlinear Granger causality test on raw series}

In order to test the nonlinear causality between efficiency and multifractality degree of the WTI crude oil market, we use nonlinear Granger causality test proposed by Diks \& Panchenko (2006).The series should be stationary time series when using Diks-Panchenko nonlinear test method. The middle part of Table 5 gives the nonlinear Granger causality test results between efficiency and multifractality degree of returns. It shows that the test results between EI4 and MD4 are similar to the results of linear Granger causality test, that is, there exist bidirectional nonlinear Granger causalities between EI4 and MD4 under the 1\% significance level. However, the test results of series are contrary to the results of linear Granger causality test: there exist bidirectional nonlinear Granger causalities between $E I 1$ and $M D 1$ under the $1 \%$ significance level.

Table 5. Granger causality results.

\begin{tabular}{|c|c|c|c|c|c|c|c|}
\hline \multirow{2}{*}{\multicolumn{2}{|c|}{ Pair }} & \multirow{2}{*}{\multicolumn{2}{|c|}{$\frac{\text { Linear causality test }}{\text { Raw data }}$}} & \multicolumn{4}{|c|}{ Nonlinear causality test } \\
\hline & & & & \multicolumn{2}{|c|}{ Raw data } & \multicolumn{2}{|c|}{ VAR } \\
\hline$X$ & $Y$ & $X \rightarrow Y$ & $Y \rightarrow X$ & $X \rightarrow Y$ & $Y \rightarrow X$ & $X \rightarrow Y$ & $Y \rightarrow X$ \\
\hline EIr4 & $M D r 4$ & $* * *$ & $* * *$ & $* * *$ & $* * *$ & $* *$ & \\
\hline$E I r 1$ & $M D r 1$ & & & $* * *$ & $* * *$ & $* * *$ & $* * *$ \\
\hline
\end{tabular}

Note: (1) $X \rightarrow Y$ denotes that $X$ does not Granger cause $Y$. (2) Notations *, ** and *** mean statistical significant under $10 \%, 5 \%$ and $1 \%$ respectively, $l_{\mathrm{X}}=l_{y}=1$. (3) The lag lengths of VAR are chosen by the Schwarz information criterion (SCI).

\subsection{Nonlinear Granger causality test on VAR-filtered residuals}

The results from the previous two steps suggest that there are some significant and persistent linear and nonlinear causal linkages between the indices However, even though we found nonlinear causality, the Diks-Panchenko test should be reapplied to filtered VAR-residuals to ensure that any causality found is strictly nonlinear in nature.

The third part of Table 5 gives the nonlinear Granger causality test results between two VAR-filtered residuals of efficiency index and multifractality degree. From these results, there is only a unidirectional nonlinear Granger causality from EI4 and MD4 for both returns. However, there exist bidirectional nonlinear Granger causalities between EI1 and $M D 1$ under the $1 \%$ significance level.

Note that there are no linear correlations between efficiency index and multifractality degree for 1 year time-window, as well as linear Granger causality in any direction, therefore the nonlinear correlation test results between efficiency index and multifractality degree are basically consistent before and after filtered by VAR.

To sum up, we only find the evidence of the nonlinear impact from efficiency index to multifractality degree for 4 year time-window, while mutual nonlinear influences between two series for 1 year time-window.

\section{Conclusion}


The exploration of the association between multifractlity degree and market efficiency of international crude oil market is a topic worthy of careful study. Our paper is the first one to discover the link between efficiency and multifractlity of international crude oil market from both linear and nonlinear aspect. Our result shows that the nonlinear relationships between efficiency and multifractlity of crude oil returns can be better revealed based on 1 year time-window. That is, for the WTI crude oil returns, the efficiency indices are always positively cross-correlated with multifractality degrees. Multifractality degrees are affected by efficiency indices in a nonlinear way and the efficiency indices are also affected by multifractality degrees in a nonlinear way.

The smaller efficiency index implies the higher degree of market efficiency. Therefore, the market efficiency and multifractality of the WTI crude oil returns interact negatively in a nonlinear mechanism. That is, the increase of multifractality degree of the WTI crude oil market will lead to the lower degree of market efficiency and vice versa. This conclusion is consistent with the correlation direction of the static analysis for a broad range of stock markets by Zunino et al. (2008) "higher multifractality is associated with a less developed (efficiency) market", and further shows that efficiency and multifractality of crude oil market influence each other with a nonlinear mechanism.

Given the results of the relations between multifratality and efficiency of crude oil market, it would be in the interest of different market participants, from investors that search for profit opportunities, to policy makers that should adjust public budget according to different conditions.

\section{Acknowledgement}

The authors were supported by the National Natural Science Foundation of China (71171108, 71071071), the Humanities and Social Science Project of Ministry of Education of China (12YJAZH020, 09YJA7909199), the Project Funded by the Priority Academic Program Development of Jiangsu Higher Education Institutions (PAPD), the Project Funded by Jiangsu Modern Service Institute (PMS) and the Science Foundation of Nanjing University of Finance \& Economics (A2010017).

\section{References}

Adrangi, B., Chatrath A., Dhanda K.K. Raffiee, K., 2001. Chaos in oil prices? Evidence from futures markets. Energy Economics 23, 405-425.

Alvarez-Ramirez, J., Cisneros, M., Ibarra-Valdez, C., Soriano A., 2002. Multifractal Hurst analysis of crude oil prices. Physica A 313, 651-670.

Alvarez-Ramirez, J., Alvarez, J., Rodriguez, E., 2008. Short-term predictability of crude oil markets: a detrended volatility analysis approach. Energy Economics 30, 2645-2656.

Alvarez-Ramirez, J., Alvarez, J., Solis, R., 2010. Crude oil market efficiency and modeling: Insights from the multiscaling autocorrelation pattern. Energy Economics 32, 993-1000.

Baek, E., Brock, W., 1992. A General Test for Non-linear Granger Causality: Bivariate Model, Working Paper. Iowa State University and University of Wisconsin, Madison,WI.

Barkoulas J., Chakraborty A., Ouandlous A., 2012. A metric and topological analysis of determinism in the crude oil spot market. Energy Economics 34, 584-591.

Diks, C., Panchenko, V., 2006. A new statistic and practical guidelines for nonparametric Granger causality testing. Journal of Economic Dynamics \& Control 30, 1647-1669.

Fama, E.F., 1970. Efficient capital markets: a review of theory and empirical work. Journal of 
Finance 25, 383-417.

Gu R.B., Shao Y.M., Wang Q.N., 2013. Is the efficiency of stock market correlated with the multifractality? An evidence from Shanghai stock market, Physica A 392: 361-370.

Hiemstra, C., Jones, J.D., 1994. Testing for linear and nonlinear Granger causality in the stock price-volume relation. Journal of Finance 49, 1639-1664.

Hurst, H.E., 1951. Long term storage capacity of reservoirs, Transactions American Society of Civil Engineers 116, 770-808.

Kantelhardt, J., Zschiegner, S., Koscielny-Bunde, E., Bunde, A., Havlin, S., Stanley, E., 2002. Multifractal detrended fluctuation analysis of nonstationary time series. Physica A 316, 87114.

Martina, E., Rodriguez, E., Escarela-Perez, R., Alvarez-Ramirez, J., 2011. Multiscale entropy analysis of crude oil price dynamics. Energy Economics 33, 936-947.

Ortiz-Cruz A., Rodriguez E., Ibarra-Valdez, Alvarez-Ramirez, J., 2012. Efficiency of crude oil markets : Evidences from informational entropy analysis. Energy Policy 41, 365-373.

Panas E., Ninni V., 2000. Are oil markets chaotic? A non-linear dynamic analysis. Energy Economics 22, 549-568.

Peng, C.K., Buldyrev, S.V., Havlin, S., Simons, M., Stanley, H.E., Goldberger, A.L., 1994. Mosaic organization of DNA nucleotides. Physics Review E 49, 1685-1689.

Tabak, B.M., Cajueiro, D.O., 2007. Are the crude oil markets becoming weakly efficient over time? A test for time-varying long-range dependence in prices and volatility. Energy Economics 29, 28-36.

Wang Y.D., Liu L., 2010. Is WTI oil market becoming weakly efficient over time? New evidence from multiscale analysis based on detrended fluctuation analysis, Energy Economics 32, 987992.

Wang Y.D., Wei Y., Wu C.F., 2010. Cross-correlations between Chinese A-share and B-share markets, Physica A 389, 5468-5478.

Zebende G.F., 2010. DCCA cross-correlation coefficient: Quantifying level of cross-correlation, Physica A 390, 614-618.

Zunino, L., Tabak, B.M., Figliola, A., Perez, D.G., Garavaglia, M., Rosso, O.A., 2008. A multifractal approach for stock market inefficiency. Physica A 387, 6556-6566. 